\title{
Concomitant diagnosis of immune deficiency and Pseudomonas sepsis in a 19 month old with ecthyma gangrenosum by host whole-genome sequencing
}

\author{
Erica Sanford, ${ }^{1,2}$ Lauge Farnaes, ${ }^{1,3}$ Serge Batalov, ${ }^{1}$ Matthew Bainbridge, ${ }^{1}$ \\ Susan Laubach, ${ }^{4} \mathrm{H}$. Michael Worthen, ${ }^{2}$ Mari Tokita, ${ }^{1}$ Stephen F. Kingsmore, ${ }^{1}$ \\ and John Bradley ${ }^{3}$ \\ ${ }^{1}$ Rady Children's Institute of Genomic Medicine, San Diego, California 92123, USA; ${ }^{2}$ Division \\ of Pediatric Critical Care Medicine, Department of Pediatrics, UCSD, San Diego, California 92093, USA; \\ ${ }^{3}$ Division of Infectious Disease, Department of Pediatrics, UCSD, San Diego, California 92093, USA; \\ ${ }^{4}$ Division of Allergy and Immunology, Department of Pediatrics, UCSD, San Diego, California 92093, USA
}

Abstract X-linked agammaglobulinemia (XLA, OMIM\#300300) is a rare monogenic primary immunodeficiency caused by mutations in the Bruton tyrosine kinase (BTK) gene. XLA is characterized by insufficient immunoglobulin levels and susceptibility to life-threatening bacterial infections. We report on a patient that presented with ecthyma gangrenosum and septicemia. Rapid trio whole-genome sequencing (rWGS) revealed an apparently de novo hemizygous pathogenic variant (c.726dupT; p.lle243TyrfsTer15) in the BTK gene. Metagenomic analysis of rWGS sequences that did not align to the human genome revealed 770 aligned to the Pseudomonas aeruginosa PAO1 genome. The patient was diagnosed with XLA and pseudomonal sepsis.

Corresponding author: esanford@ucsd.edu

(c) 2018 Sanford et al. This article is distributed under the terms of the Creative Commons Attribution-NonCommercial License, which permits reuse and redistribution, except for commercial purposes, provided that the original author and source are credited.

Ontology terms: congenital neutropenia; immune dysregulation; sepsis

Published by Cold Spring Harbor Laboratory Press

doi: $10.1101 / \mathrm{mcs} . a 003244$

\section{CASE PRESENTATION}

A 19-mo-old twin Hispanic male was admitted to the hospital with fever $\left(38.5^{\circ} \mathrm{C}\right)$ and compensated septic shock. Physical examination revealed approximately one dozen deep, indurated purpuric plaques with necrotic changes on the extremities and buttocks (Fig. 1). The patient's twin brother had died of septic shock, purpura fulminans, and disseminated intravascular coagulation in an outside hospital $1 \mathrm{~d}$ prior, after a 2-d hospitalization for similar symptoms. The twin brothers were born at 36-wk gestation and had not previously been hospitalized, but the mother reported that for the past 8 mo they had been frequently ill with respiratory tract infections requiring multiple courses of antibiotics. At the time of admission, the patient's weight was $12 \mathrm{~kg}$, which fell into the 70th percentile for age-matched male controls. There was no family history of immunodeficiency.

Laboratory evaluation was significant for a white blood cell count of $2800 \mathrm{~L}^{-1}$ (reference $6000-14000$ ) with $50 \%$ monocytes (2\%-12\%), 32\% lymphocytes (30\%-60\%), $10 \%$ atypical lymphocytes (0\%-10\%), $2 \%$ segmented neutrophils (45\%-70\%), and $7 \%$ band neutrophils $(0 \%-10 \%)$, C-reactive protein (CRP) level of $30.10 \mathrm{mg} / \mathrm{dl}(0.00-0.99)$, an erythrocyte 
COLD SPRING HARBOR Molecular Case Studies
WGS diagnosis of immunodeficiency and sepsis
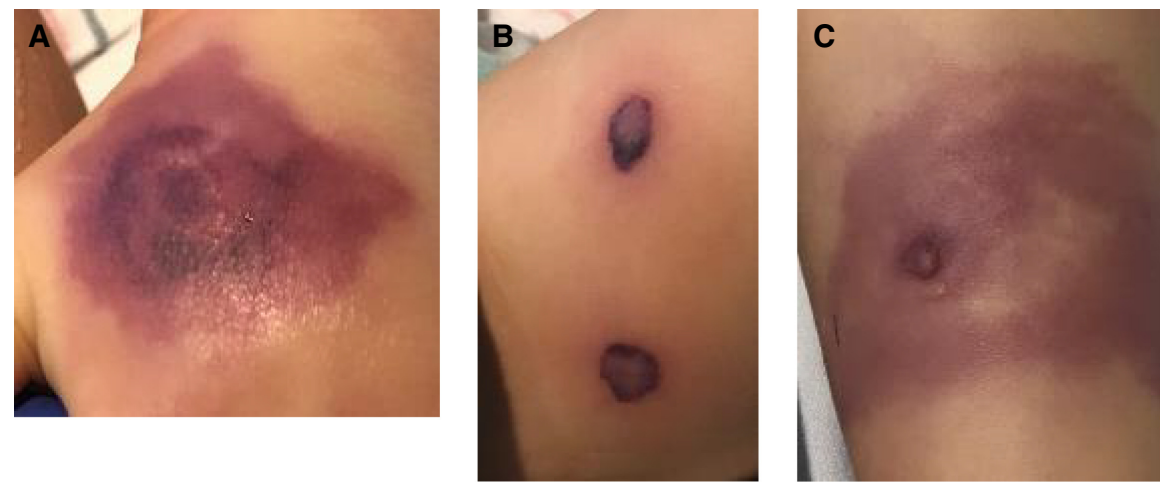

Figure 1. Skin lesions in the affected patient showing $(A)$ left hand, $(B)$ left thigh, and $(C)$ right lower leg pseudomonal ecthyma gangrenosum.

sedimentation rate of $5 \mathrm{~mm} / \mathrm{h}(0-10)$, procalcitonin level of $49.04 \mathrm{ng} / \mathrm{ml}(<2.0)$, prothrombin time (PT) of $34.7 \mathrm{sec}(11.4-14)$, and an international normalized ratio (INR) of 3.4 (0.9-1.2). Immunoglobulin levels were lgG $<135 \mathrm{mg} / \mathrm{dl}(413-1112), \operatorname{lgA}=32 \mathrm{mg} / \mathrm{dl}(21-117)$, and $\lg \mathrm{M}=19 \mathrm{mg} / \mathrm{dl}(30-146)$.

The patient was started on vancomycin, ceftriaxone, doxycycline, and gentamicin, as the identity of the causative organism for this presumed community-acquired, life-threatening infection was not initially known. Cultures from the patient's blood were sterile. Filgrastim (G-CSF) was also administered subcutaneously at a dose of $5 \mathrm{mcg} / \mathrm{kg}$ daily for an absolute neutrophil count of $252 \mu \mathrm{l}$ in the setting of overwhelming infection. Within the first $24 \mathrm{~h}$ of admission, the patient required intubation for respiratory failure as well as vasopressor therapy with multiple medications for fluid-refractory hypotension and poor perfusion. Because of concerns about hypercoagulability, intravenous immunoglobulin (IVIG) was initially held, but as the patient's condition worsened, a dose of $0.4 \mathrm{mg} / \mathrm{kg}$ of IVIG was given, which increased the patient's IgG level to $646 \mathrm{mg} / \mathrm{dl}$.

A tissue culture obtained from a 5-mm punch biopsy of a cutaneous lesion grew Pseudomonas aeruginosa on the third hospital day. At that point, meropenem was added to the regimen and other antibiotics were eventually stopped. The diagnosis of pseudomonal ecthyma gangrenosum in this patient and the presumptive identical diagnosis in his deceased twin brother were highly suspicious for a primary immune deficiency, but the combination of neutropenia and low lgG and lgM levels did not conclusively point to one diagnosis. A lymphocyte enumeration panel was sent and resulted a CD19 absolute count of $<20$ cells/ $\mu$ l (830-1880) the following day, consistent with absent $B$ cells. Given the patient's critically ill state and the recent death of his twin, rapid trio whole-genome sequencing (rWGS) was undertaken simultaneously on the patient and his parents. In just more than $72 \mathrm{~h}$, on the fourth hospital day, an apparently de novo hemizygous pathogenic variant (c.726dupT; p.lle243TyrfsTer15; Table 1) in the BTK gene was detected and the patient was diagnosed with X-linked agammaglobulinemia (XLA, OMIM\#300300). As a result, filgrastim injections were discontinued and IVIG was administered to maintain IgG levels of $>800 \mathrm{mg} / \mathrm{dl}$. On the same day (hospital day 4), verbal communication from the hospital where the patient's brother expired confirmed a positive blood culture for pseudomonas in the deceased twin. The patient's clinical status and level of cardiorespiratory support slowly improved on anti-pseudomonal antibiotics and IVIG therapy. He required regular debridement of the gangrenous lesions by plastic surgery and eventually underwent skin grafting. He was discharged from the hospital after a total of 5 mo. 


\begin{tabular}{|c|c|c|c|c|c|c|}
\hline Gene & $\begin{array}{l}\text { Genomic } \\
\text { location }\end{array}$ & HGVS cDNA & HGVS protein & Zygosity & $\begin{array}{l}\text { Parent } \\
\text { of origin }\end{array}$ & $\begin{array}{c}\text { Variant } \\
\text { interpretation }\end{array}$ \\
\hline BTK & $\begin{array}{l}\text { NC_000023.10: } \\
\text { Chr } \\
\text { X:100615605 } \\
\text { (GRCh37/ } \\
\text { hg19) }\end{array}$ & $\begin{array}{l}\text { NM_000061.2: } \\
\text { c.726dupT }\end{array}$ & $\begin{array}{l}\text { NP_000052.1 } \\
\text { p.lle243TyrfsTer15 }\end{array}$ & Hemizygous & De novo & Pathogenic \\
\hline
\end{tabular}

\section{TECHNICAL ANALYSIS AND METHODS}

The patient was enrolled in an IRB-approved research study. Blood was drawn immediately following consent for trio rWGS. The WGS testing was performed in a CAP/CLIA environment. DNA was subsequently extracted and sequenced on a HiSeq 4000 (Illumina). Rapid alignment and nucleotide variant calling was performed using the Dragen (Edico Genome) hardware and software (Miller et al. 2015). Proband and parental samples were sequenced to a mean coverage of $40 \times$. Variants were annotated and analyzed in Opal Clinical (Fabric Genomics) (Coonrod et al. 2013). Initially, variants were filtered to retain those with allele frequencies of $<1 \%$ in the Exome Variant Server, 1000 Genomes Samples, and Exome Aggregation Consortium database (http://evs.gs.washington.edu/EVS/2016; Karczewski et al. 2017). A gene panel was built in Phenolyzer (Yang et al. 2015) using Human Phenotype Ontology (HPO) (Köhler et al. 2016). This panel included 1098 genes related to the following HPO terms: immunodeficiency (HP:0002721), neutropenia (HP:0001875), and sepsis (HP:0100806). Variants were further filtered to retain those mapping to these 1098 genes yielding 1068 proband calls (465 homozygous variants, 509 heterozygous inherited variants, and 15 heterozygous de novo variants). Manual curation revealed one variant as pathogenic by ACMG guidelines (Richards et al. 2015). Specifically, the criteria invoked to score this variant as pathogenic included PVS1 (null variant in a gene where loss of function is a known mechanism of disease), PS2 (de novo variant), and PM2 (variant is absent from controls). This variant was confirmed by Sanger sequencing. The diagnosis was made by rWGS in $4 \mathrm{~d}$.

\section{VARIANT INTERPRETATION}

This patient was found to be hemizygous for an apparently de novo known pathogenic frameshift variant (c.726dupT; p.lle243TyrfsTer15) in the Bruton tyrosine kinase (BTK) gene. Pathogenic variants in BTK have been implicated in XLA (OMIM\#300300).

The de novo p.lle243TyrfsTer15 pathogenic variant has been previously reported in a single individual with XLA (Holinski-Feder et al. 1998). p.lle243TyrfsTer15 is absent from the ExAC and gnomAD population databases. The deletion causes a frameshift starting with codon Isoleucine 243, changes this amino acid to a tyrosine residue, and creates a premature stop codon at position 15 of the new reading frame. This pathogenic variant is predicted to cause loss of normal protein function through protein truncation or nonsense-mediated decay of the mRNA transcript.

\section{METAGENOMICS}

Per institutional protocol, samples were processed via a standard clinically validated workflow, in addition to passing a battery of quality control (QC) scripts (Farnaes et al. 2018). 
COLD SPRING HARBOR Molecular Case Studies
WGS diagnosis of immunodeficiency and sepsis

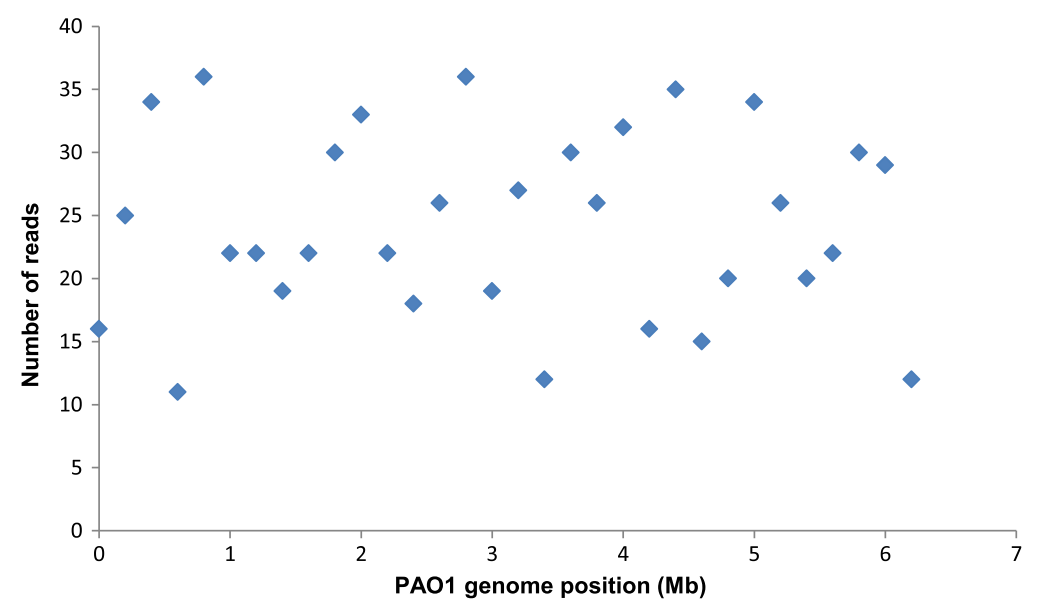

Figure 2. Uniformly random spatial distribution of 770 reads from the proband's DNA sample (in which the $y$ axis represents the number of reads) aligned to the PAO1 genome (in which the $x$-axis is position Mb of the PAO1 genome; total size $=6.2 \mathrm{Mb}$ ).

Using the reference human genome hg37dh, the $\mathrm{QC}$ metric of percent aligned reads passed at $98.8 \%$ and did not have a statistically significant deviation from median of past samples $(N=100)$. Given the pseudomonas sepsis phenotype, a metagenomics exploration was performed: After isolating the unaligned portion of reads, the most frequent, randomly sampled 500 reads were checked against the NCBI NR database, and significant similarity to $P$. aeruginosa genome was noted, whereas similarity to other pathogenic or nonpathogenic genomes was not found. The random sampling was drawn from the 500 most abundant reads (including duplicates). Then, all unaligned reads were aligned to reference $P$. aeruginosa PAO1 genome and 770 paired reads (654 unique; duplication rate 14\%) were aligned. The qualities of the PAO1-aligned reads were not significantly different from those that aligned to the reference human genome (mean $Q$ in read $1=36.28$ and in read $2=36.04$, aligned to PAO1; mean $Q$ in read $1=36.09$ and in read $2=35.66$, aligned to hg19). For negative controls, unaligned portions of the patient's parental genomic DNA as well as genomic DNA from five random, unrelated persons were aligned to the reference PAO1 genome and 0-3 paired reads aligned in each (establishing the level of random alignments). Furthermore, the pattern of spatial distribution of the 770 reads from the proband's DNA sample to PAO1 genome was nearly uniform over the full length of the PAO1 genome $(6.2 \mathrm{Mb}, 25 \pm 10$ reads in each $200 \mathrm{~kb}$ window, i.e., $\sim 1 \mathrm{read} / 8 \mathrm{~kb}$ ), resulting in the conclusion of the bona fide lowtiter presence of $P$. aeruginosa in the proband's DNA sample (Fig. 2).

\section{DISCUSSION}

Inherited defects of the immune system have an incidence of nearly 1 in 2000 children (Bonilla et al. 2015). More than 300 primary immunodeficiencies (PIDs) are currently known, with the list rapidly expanding (Bonilla et al. 2015; Picard et al. 2015). Between 2013 and 2015 alone, 34 new gene defects for PID were identified (Picard et al. 2015). The severity of infection in affected patients encompasses a spectrum ranging from recurrent respiratory illnesses to life-threatening systemic disease. Current consensus guidelines advocate for a stepwise approach to identifying a suspected PID (Bonilla et al. 2015). However, identifying specific immunodeficiencies in this manner can take weeks to months and delay in diagnosis is significantly associated with increased mortality in these patients (Joshi et al. 2009). More 
recently, whole-exome sequencing has started to be explored as a high-throughput approach to diagnosing patients with suspected PIDs, with a diagnostic rate of up to $40 \%$ (Stray-Pedersen et al. 2017). rWGS is emerging as a technology capable of making a specific genetic diagnosis in time to effect changes in acute clinical management. In our patient, the diagnosis of XLA was highly likely after the lymphocyte enumeration panel demonstrated absent $B$ cells, and the next traditional step in the absence of rWGS would be to sequence the suspected gene (in this case, BTK), which takes several weeks to result. rWGS was able to make the diagnosis of XLA within $74.5 \mathrm{~h}$.

$\mathrm{XLA}$ is a primary humoral immunodeficiency typically characterized by severe hypogammaglobinemia and recurrent bacterial infections (Smith and Berglöf 2016). It is caused by mutations in BTK, which encodes Bruton tyrosine kinase (Btk) (Smith and Berglöf 2016). Btk is a signal transduction molecule essential to B-cell lineage development, and its loss impairs the progression of pre-B cells to mature lymphocytes (Ochs and Smith 1996). The lack of mature $B$ lymphocytes results in insufficient immunoglobulin levels, and affected patients are unable to mount an appropriate antibody response to infection. Affected persons have normal levels of $\mathrm{gg}$ at birth because of transplacental passage of maternal immunoglobulin, but become susceptible to bacterial and enteroviral infections as these levels gradually wane during the first year of life (Ochs and Smith 1996). The diagnosis of XLA is suspected when laboratory investigations demonstrate absent $B$ cells and a reduction in all classes of immunoglobulins, and it is confirmed by identifying a pathogenic variant in BTK (Ochs and Smith 1996; Smith and Berglöf 2016).

The bacterial infections responsible for causing severe disease in patients with XLA are most frequently the pyogenic organisms Streptococcus pneumoniae and Hemophilus influenzae (Zenone and Souillet 1996; Winkelstein et al. 2006). Although uncommon, in addition to our patient there have been case reports of patients presenting with $P$. aeruginosa septicemia and ecthyma gangrenosum that were subsequently diagnosed with XLA (Nussinovitch et al. 1991; Zenone and Souillet 1996).

The diagnosis of XLA by rWGS explained the patient's susceptibility to pseudomonal sepsis, indicated the appropriate management to be regular IVIG infusions, and may have prevented additional life-threatening infections. Retrospectively, it also provided an explanation for the death of his twin brother. The patient will be closely followed by immunology for the duration of his lifetime. In addition to monthly IVIG infusions, the patient will have to avoid live vaccinations and his treating physicians should have a low threshold for initiating antibiotic therapy when he is ill.

The metagenomic analysis identification of $770 P$. aeruginosa genome sequences confirmed the presence of the bacterial DNA in the patient's bloodstream (DNAemia) and the clinical diagnosis of severe sepsis. Blood cultures in our patient, while receiving broad spectrum antibiotics, never grew Pseudomonas, and the bacterium was identified by culture of a skin biopsy of one of the gangrenous lesions. Patients with bacterial sepsis infrequently have skin lesions available for biopsy_-in the absence of that sample for this patient, nucleic acid analysis would potentially have been the only acute method for identifying the responsible bacterial agent. This raises the possibility that rWGS could potentially have utility in identification of disease-causing organisms, especially in cases of culture-negative sepsis, and warrants further exploration.

\section{SUMMARY}

We report on a case of XLA presenting as pseudomonal ecthyma gangrenosum and severe sepsis that was diagnosed expeditiously by rWGS. rWGS identified a de novo frameshift variant (c.726dupT; p.lle243TyrfsTer15) in BTK, the Bruton tyrosine kinase gene on 
COLD SPRING HARBOR Molecular Case Studies
WGS diagnosis of immunodeficiency and sepsis

\section{Referees}

Dustin Baldridge

Jim Connelly

Eric W. Klee

Competing Interest Statement

The authors have declared no competing interest.

Received July 25, 2018; accepted in revised form September 24, 2018.
Chromosome $X$. Thus, the proband was determined to be hemizygous for the monogenic disorder XLA. Additionally, metagenomic analysis of rWGS sequences that did not align to the human genome were found to align to the $P$. aeruginosa PAO1 genome.

\section{ADDITIONAL INFORMATION}

\section{Data Deposition and Access}

The variant was submitted to ClinVar (http://www.ncbi.b/nih.n/m.gov/clinvar) and can be found under accession number SCV000809043.

\section{Ethics Statement}

Informed and signed consent forms were obtained for all sequenced individuals in this study. The project is approved by the Institutional Review Board of the University of California at San Diego under protocol \#160468 and has received nonsignificant risk status in a preInvestigational Device Exemption submission to the Food and Drug Administration.

\section{Author Contributions}

E.S. prepared the manuscript and performed the phenotyping; L.F., S.F.K., and J.B. supervised and prepared the manuscript; S.B., M.B., and M.T. performed the variant interpretation and supervised the analysis; and S.L. and H.M.W. performed the clinical implementation. All authors contributed to the reviewing of the final version.

\section{Funding}

Supported by Rady Children's Hospital, National Institute of Child Health and Human Development, and National Human Genome Research Institute (grant U19HD077693).

\section{REFERENCES}

Bonilla FA, Khan DA, Ballas ZK, Chinen J, Frank MM, Hsu JT, Keller M, Kobrynski LJ, Komarow HD, Mazer B. 2015. Practice parameter for the diagnosis and management of primary immunodeficiency. J Allergy Clin Immunol 136: 1186-1205.

Coonrod EM, Margraf RL, Russell A, Voelkerding KV, Reese MG. 2013. Clinical analysis of genome next-generation sequencing data using the Omicia platform. Expert Rev Mol Diagn 13: 529-540.

Farnaes L, Hildreth A, Sweeney NM, Clark MM, Chowdhury S, Nahas S, Cakici J, Bensen W, Kaplan R, Kronick $\mathrm{R}$, et al. 2018. Rapid whole-genome sequencing decreases infant morbidity and cost of hospitalization. NPJ Genom Med 3: 10.

Holinski-Feder E, Weiss M, Brandau O, Jedele KB, Nore B, Bäckesjö CM, Vihinen M, Hubbard SR, Belohradsky $\mathrm{BH}$, Smith $\mathrm{Cl}$, et al. 1998. Mutation screening of the BTK gene in 56 families with X-linked agammaglobulinemia (XLA): 47 unique mutations without correlation to clinical course. Pediatrics 101: 276-284.

Joshi AY, lyer VN, Hagan JB, St. Sauver JL, Boyce TG. 2009. Incidence and temporal trends of primary immunodeficiency: a population-based cohort study. Mayo Clin Proc 84: 16-22.

Karczewski KJ, Weisburd B, Thomas B, Solomonson M, Ruderfer DM, Kavanagh D, Hamamsy T, Lek M, Samocha KE, Cummings BB, et al. 2017. The ExAC browser: displaying reference data information from over 60000 exomes. Nucleic Acids Res 45: D840-D845.

Köhler S, Vasilevsky NA, Engelstad M, Foster E, McMurry J, Aymé S, Baynam G, Bello SM, Boerkoel CF, Boycott KM, et al. 2016. The Human Phenotype Ontology in 2017. Nucleic Acids Res 45: D865-D876.

Miller NA, Farrow EG, Gibson M, Willig LK, Twist G, Yoo B, Marrs T, Corder S, Krivohlavek L, Walter A, et al. 2015. A 26-hour system of highly sensitive whole genome sequencing for emergency management of genetic diseases. Genome Med 7: 100.

Nussinovitch M, Frydman M, Cohen HA, Varsano I. 1991. Congenital agammaglobulinemia presenting with ecthyma gangrenosum. Acta Paediatr Scand 80: 732-734. 
Ochs HD, Smith CE. 1996. X-linked agammaglobulinemia a clinical and molecular analysis. Medicine 75: 287-299.

Picard C, Al-Herz W, Bousfiha A, Casanova JL, Chatila T, Conley ME, Cunningham-Rundles C, Etzioni A, Holland SM, Klein C, et al. 2015. Primary immunodeficiency diseases: an update on the classification from the International Union of Immunological Societies Expert Committee for primary immunodeficiency. J Clin Immunol 35: 696-726.

Richards S, Aziz N, Bale S, Bick D, Das S, Gastier-Foster J, Grody WW, Hegde M, Lyon E, Spector E, et al. 2015. Standards and guidelines for the interpretation of sequence variants: a joint consensus recommendation of the American College of Medical Genetics and Genomics and the Association for Molecular Pathology. Genet Med 17: 405-424.

Smith CE, Berglöf A. 2016. X-linked agammaglobulinemia. In GeneReviews ${ }^{\circledR}$ [Internet] (ed. Adam MP, Ardinger HH, Pagon RA, Wallace SE, Bean LJH, Stephens K, Amemiya A). University of Washington, Seattle. https://www.ncbi.nlm.nih.gov/books/NBK1453

Stray-Pedersen A, Sorte HS, Samarakoon P, Gambin T, Chinn IK, Coban Akdemir ZH, Erichsen HC, Forbes LR, Gu S, Yuan B, et al. 2017. Primary immunodeficiency diseases: genomic approaches delineate heterogeneous Mendelian disorders. J Allergy Clin Immunol 139: 232-245.

Winkelstein JA, Marino MC, Lederman HM, Jones SM, Sullivan K, Burks AW, Conley ME, Cunningham-Rundles C, Ochs HD. 2006. X-linked agammaglobulinemia: report on a United States registry of 201 patients. Medicine 85: 193-202.

Yang H, Robinson PN, Wang K. 2015. Phenolyzer: phenotype-based prioritization of candidate genes for human diseases. Nat Methods 12: 841-843.

Zenone T, Souillet G. 1996. X-linked agammaglobulinemia presenting as Pseudomonas aeruginosa septicemia. Scand J Infect Dis 28: 417-418. 


\section{COLD SPRING HARBOR Molecular Case Studies}

\section{Concomitant diagnosis of immune deficiency and Pseudomonas sepsis in a 19 month old with ecthyma gangrenosum by host whole-genome sequencing}

Erica Sanford, Lauge Farnaes, Serge Batalov, et al.

Cold Spring Harb Mol Case Stud 2018, 4: a003244

Access the most recent version at doi: $10.1101 / \mathrm{mcs} . a 003244$

\begin{tabular}{cl}
\hline License & $\begin{array}{l}\text { This article is distributed under the terms of the Creative Commons } \\
\text { Attribution-NonCommercial License, which permits reuse and redistribution, except } \\
\text { for commercial purposes, provided that the original author and source are credited. }\end{array}$ \\
Email Alerting & $\begin{array}{l}\text { Receive free email alerts when new articles cite this article - sign up in the box at the } \\
\text { top right corner of the article or click here. }\end{array}$ \\
\hline
\end{tabular}

